\title{
The effects of Pavlovian CSs on two food-reinforced baselines- with and without noncontingent shock
}

\author{
THOMAS S. HYDE \\ Case Western Reserve University, Cleveland, Ohio 44106
}

\begin{abstract}
Two experiments explored the effects of Pavlovian (tone-shock) CS,$+ \mathrm{CS}-$, and truly random control (TRC) contingencies on two different food-reinforced instrumental baselines. One foodreinforced baseline contained noncontingent shock, while the other did not. In the first experiment, a TRC contingency was shown to produce suppression of food-reinforced responding, while a CScontingency did not. When noncontingent shock was added to the baseline, however, the TRC stimuli failed to produce suppression, and the CS- contingency increased response rates over baseline level. In a second experiment, the effects of TRC and CS+ contingencies were compared on these same two baselines. While the CS+ produced suppression on both shock and no-shock baselines, the TRC contingency again produced suppression on only the no-shock baseline.
\end{abstract}

Rescorla $(1967,1972)$ has suggested a view of Pavlovian conditioning which argues that the acquisition of a CR is primarily a function of the degree of contingency between the CS and US, or the degree to which the CS predicts the occurrence of the US. An important feature of this analysis is the prominent role it gives to negative contingencies, i.e., where the CS predicts the nonoccurrence of the US. Bull and Overmier (1968), Hyde (1976), and Rescorla and LoLordo (1965), for example, have all shown reliable effects of both positive and negative contingencies.

A major implication of Rescorla's contingency view has been the question of proper control procedures in Pavlovian conditioning. Perhaps the most widely used control procedure has been one in which subjects are exposed to both the CS and US in the same situation, but in such a way that they are never paired. Rescorla (1967) has pointed out that from his contingency view of conditioning, such an "explicitly unpaired" control would introduce a negative contingency, and has suggested that a more appropriate control is one in which the CS and US are presented with no temporal contingency, i.e., the CS predicts neither the presence nor the absence of the US. Rescorla refers to such a procedure as a "truly random control" (TRC).

While there has been little argument that different contingencies between Pavlovian CSs and USs produce different behavioral effects, there has been disagreement over what these differences reflect. In the conditioned suppression paradigm, for example,

Requests for reprints may be sent to Thomas S. Hyde, Department of Psychology, Mather Memorial Building, Case Western Reserve University, Cleveland, Ohio 44106. there is ample evidence that stimuli predictive of aversive USs (CS + ), stimuli predictive of the absence of USs (CS-), and stimuli which predict neither the presence nor absence of the US (TRC stimuli) produce clear behavioral differences. The primary controversy has centered around whether the TRC stimulus is excitatory or neutral and/or whether the CS- is neutral or inhibitory. Evidence consistent with both views has been reported, and has come not from relative differences in the effects of these contingencies, but rather from their absolute effects relative to some baseline. When differences between TRC stimuli, CS +s, and/or CS-s are found, and when the TRC stimuli seem to produce some type of behavioral effect relative to the baseline (e.g., Benedict \& Ayres, 1972; Kremer \& Kamin, 1971; Quinsey, 1971), the differences are taken as evidence that the TRC stimuli are excitatory (because of chance pairings with the US) and the CS-s are neutral. When similar differences are found but the TRC stimuli do not seem to produce a behavioral effect relative to the baseline (e.g., Bull \& Overmier, 1968; Rescorla, 1968, 1969), differences are taken for a contingency view, i.e., that the TRC stimuli are associatively neutral and the CS-s are inhibitory.

Sheldon (1973) has pointed out that because there is no way of knowing the relative contribution of associative and nonassociative factors to the behavioral effects produced by different contingencies, it is impossible to support either a contingency or a more traditional "pairings" view of conditioning from the results of these experiments. Although it is clearly an arbitrary use of Sheldon's (1973) argument, it does seem possible that those experiments which have shown behavioral effects of TRC stimuli are particularly likely to have been influenced by 
what might be called nonassociative factors. These experiments (e.g., Benedict \& Ayres, 1972; Kremer \& Kamin, 1971; Quinsey, 1971) have all used a conditioned suppression paradigm and are highly similar in design. Subjects first learned a simple instrumental response for food reinforcement. In a second conditioning phase, the response was physically prevented and an exteroceptive stimulus and an aversive US were presented with various contingencies. In a third, "reacquisition" phase, the stimulus and the aversive US no longer occurred, and the instrumental response for food was again permitted. In a final test phase, the exteroceptive stimulus was again presented, and its effects on the instrumental baseline were examined for the different CS-US contingencies.

A major problem with this experimental paradigm is that subjects undoubtedly learn a discrimination between the Pavlovian conditioning and instrumental responding phases of the experiment. The "reacquisition" phase, where the exteroceptive CSs and aversive USs are excluded and the instrumental response for reinforcement again becomes possible, would seem likely to produce such a discrimination. The gradual recovery of responding during the reacquisition phase serves as evidence that anxiety conditioned to situational cues (other than the exteroceptive CSs) is being systematically extinguished. Because of this discrimination, the occurrence of the exteroceptive stimuli during the test phase of the experiment could produce a substantial excitatory nonassociative effect by breaking down the discrimination which subjects have learned during the acquisition phase of the experiment. Even were the TRC stimuli associatively neutral, they could produce a conditioned suppression effect through this nonassociative effect alone. Also, the CS- could produce no suppression, not because it is associatively neutral, but because it is a conditioned inhibitor of anxiety and thereby fortuitously cancels out the nonassociative excitatory effect resulting in a stimulus that is behaviorally neutral.

Quinsey (1971) has presented a related argument which leads to the same general conclusions. He has argued that in TRC procedure, anxiety would be conditioned equally to the CS and all other situational cues. If the reacquisition phase systematically extinguishes anxiety which was conditioned to these situational cues, the TRC stimulus may show a suppression effect which would be mistakenly interpreted as reflecting associative conditioning. Equally important, it follows that if anxiety is extinguished to the situational cues and is not present during the test phase, the CS- would be unlikely to show any behavioral effects, even if it were a strong inhibitor of anxiety, as there would be no anxiety to inhibit.
Although the above argument is speculative, it would seem a simple matter to prevent the extinction of anxiety to situational cues and thereby prevent subjects from learning a discrimination between the conditioning and test phases of the experiment. This could be done by presenting noncontingent aversive USs during the reacquisition and testing phases of the experiment. This would appear to be a much better baseline on which to test the effects of different Pavlovian contingencies. The first experiment will compare the behavioral effects of TRC and CS - contingencies both under the experimental conditions found in previous experiments and under conditions where subjects are exposed to noncontingent shocks during the reacquisition and test phases of the experiment. If the learning of a discrimination between the conditioning and test phases of the experiment (or extinction of anxiety conditioned to situational cues) is important in increasing the likelihood of observing nonassociative effects, then the two testing conditions should produce different results. On the food-reinforced baseline without noncontingent shock, it is expected that the negative contingency $(\mathrm{CS}-$ ) will produce minimal effects on behavior, while the TRC contingency will produce the previously reported suppression effect. When extinction of anxiety to situational cues is prevented by adding noncontingent shock to the food-reinforced baseline, it is expected that the TRC contingency will not produce conditioned suppression, and that the CS- will inhibit anxiety conditioned to situational cues and raise the level of responding above baseline.

\section{EXPERIMENT I}

\section{Subjects and Apparatus \\ Method}

The subjects were 24 naive male Long-Evans rats, approximately 100 days old at the beginning of the experiment.

The experiment was conducted in two identical operant conditioning chambers, $25 \times 25 \mathrm{~cm}$ in size. The ceilings and side walls of the chambers were constructed of clear acrylic, and the front and rear walls of aluminum. On the front panel of each chamber was a food magazine and a retractable response lever. The floor of the chamber was constructed of 6-mm stainless steel bars which were connected to a $150 \mathrm{~K} \Omega$ fixed-impedence shock source, as described by Campbell and Teghtsoonian (1958). Shock was scrambled via a shock scrambler described by Hoffman and Fleshler (1962). The shock US was $0.5 \mathrm{sec}$ in duration and was nominally $1.0 \mathrm{~mA}$. The CS was a 1 -min tone $(1,000 \mathrm{~Hz})$, and was delivered through a speaker located directly above the experimental chamber. The chambers were housed in sound-attenuated boxes, with a masking noise provided by fans in each box. The experimental chambers were connected to the necessary programming and recording equipment, which was located in the next room.

\section{Procedure}

The experiment was a $2 \times 2$ factorial, in which there were two types of Pavlovian contingency during the conditioning 
phase (TRC and CS-) and two different testıng conditions (with and without noncontingent shock).

Preliminary training. All subjects were reduced to $80 \%$ of their ad-lib body weight and placed on a $23-\mathrm{h}$ feeding schedule which continued for the duration of the experiment. All subjects were given two daily sessions of adaptation to the experimental chamber, four sessions of magazine training, two sessions of continuous reinforcement for leverpressing, one session of training on a VI 30-sec schedule of reinforcement, and then 20 daily sessions of training on a Vi 2-min schedule of reinforcement. All training sessions were $40 \mathrm{~min}$ in duration, and reinforcement for leverpressing consisted of a single $45-\mathrm{mg}$ food pellet.

Pavlovian conditioning. Following the final day of instrumental training, the lever was retracted from the chamber and the subjects were randomly divided into two experimental groups of 12 subjects each. One group (TRC) had the auditory CS and the shock US presented with no temporal contingency. During each 40-min session, the 1-min CS was programmed to occur 15 times. The onsets of the 15 stimulus presentations were randomly distributed throughout the session, with the restriction that a period of at least $5 \mathrm{sec}$ separate two presentations. This procedure resulted in an interstimulus interval which averaged $1.67 \mathrm{~min}$. The 15 shock USs were also randomly distributed throughout the session. This randomization was independent of the presentation of the auditory $\mathrm{CS}_{2}$ Shocks could occur at any time, with the restriction that at least $5 \mathrm{sec}$ had to separate any two shock presentations. The probability of a CS/US pairing in the TRC condition was 0.375 . This translated into four to seven such pairings per experimental session.

The conditioning group (CS-) had the same schedule of stimulus presentations as did the TRC condition. In this group, however, the 15 shock USs were programmed to occur only during the interstimulus interval. Shocks could occur at any time during non-CS periods, given the restrictions that they must be at least $5 \mathrm{sec}$ apart and at least $5 \mathrm{sec}$ from the termination of an auditory CS. Conditioning lasted for 15 daily sessions for all subjects, and the randomized pattern of CS and US presentation was changed every three sessions, or five times during the conditioning phase of the experiment.

Reacquisition training. Following the final day of conditioning, all subjects were given a single 40 -min session, during which the response lever was again introduced into the chamber and responding produced food reinforcement on a VI 2 -min schedule. If subjects failed to respond during the $40-\mathrm{mm}$ session, they were given an additional session later in the day. Following this initial day of reacquisition training, the subjects were divided into two testing groups. One-half of the subjects from each of the two conditioning groups were randomly assigned to each of the two testing conditions. For one condition (no shock), there were 2 additıonal days of reacquisition training where responding was reinforced on a VI 2 -min schedule. In the other testing condition (shock), noncontingent shocks were introduced onto the foodreinforced baselıne. On the second day of reacquisition, two shocks were introduced, and on the third day, four shocks. Shocks occurred 10 and $30 \mathrm{~min}$ from the onset of the second session, and $10,15,30$, and $35 \mathrm{~min}$ from the onset of the third session. The intensity and duration of the shocks was identical to that for the prior conditioning phase of the experiment.

Suppression testing. The two reacquisition conditions (shock and no shock) made up the two testing conditions. Subjects who had not received shock during reacquisition received no shocks during testing, and subjects who received shocks during reacquisition continued to receive shocks during testing on the same schedule used during the final day of reacquisition.

For all subjects, the 1-min CS was presented twice during each of the four daily testing sessions. The CS was presented after 20 and $\mathbf{4 0}$ min from the onset of each training session. The subjects were removed from the experimental chamber approximately $5 \mathrm{~min}$ after the final CS presentation.
Suppression ratios were used to assess the effects of the CS on instrumental behavior. $A D /(D+B)$ suppression ratio was calculated for each subject on each test trial. In this ratio, D denotes the number of responses made during the $1-\min \mathrm{CS}$, and $\mathrm{B}$ denotes the number of responses during the 1-min period immediately preceding each CS presentation. With such a ratio, a value less than 0.50 indicates that responding during the CS was lower than that during the immediately preceding period, and a value greater than 0.50 indicates that the responding during the CS was greater.

\section{Results and Discussion}

The noncontingent shock during reacquisition and testing had a significant effect in retarding the recovery of leverpressing. Subjects in the no-shock condition recovered to $86 \%$ of their preconditioning response rate by the final day of reacquisition, while subjects in the shock condition recovered to only $47 \%$ of their preconditioning level. All subjects in the shock condition did, however, produce a steady level of responding during testing.

The effects of the TRC stimuli and CS $-s$ on the shock and no-shock baselines are presented in Figure 1. The stimuli had different effects upon responding as a result of the treatment conditions. A two-way repeated measures ANOVA (Treatment Conditions by Test Sessions) yielded a statistically significant effect for Treatment Conditions $(F=7.82$, $\mathrm{df}=3 / 20, p<.01)$. The Sessions effect and the Treatment by Sessions interaction failed to reach statistical significance. As is apparent in Figure 1, the TRC stimulus on the no-shock baseline produced a conditioned suppression effect, while the same TRC contingency on the shock baseline did not. A contrast on these two group means yielded an $F=8.93(\mathrm{df}=1 / 20, p<.01)$. The Pavlovian CS on the no-shock baseline seemed to produce little behavior effect, while the same CS - contingency

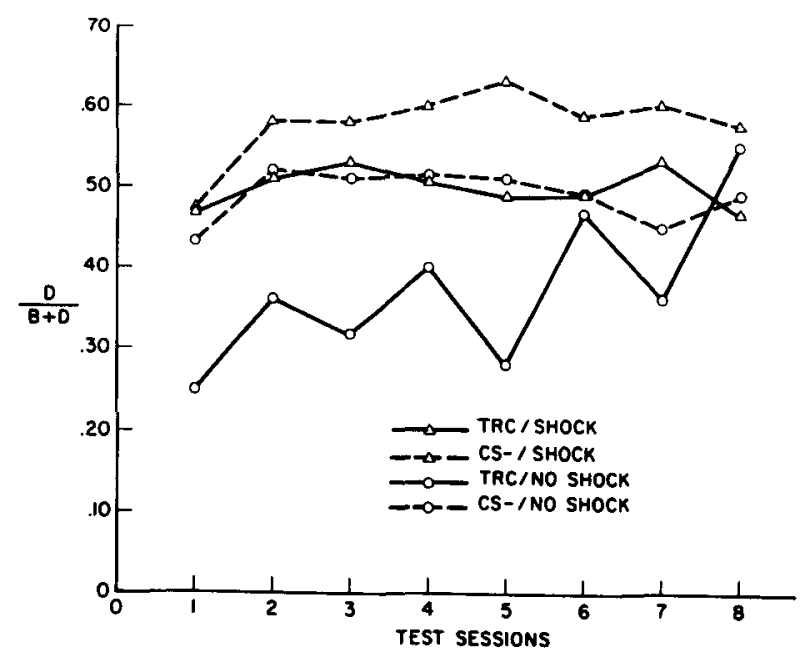

Figure 1. Mean suppression ratios for each test trial for each group in Experiment $\mathbf{I}$. 
on the shock baseline increased the response rates above the baseline level. The differences between these two $\mathrm{CS}-$ conditions produced an $\mathrm{F}=4.63$ (df $=1 / 20, p<.05)$.

If, as suggested earlier, subjects in the no-shock condition learned a discrimination between the conditioning and test phases of the experiment by having anxiety conditioned to situational cues extinguished during reacquisition, the TRC stimulus could have produced a behavior effect through nonassociative factors alone. This notion seems supported by the failure of a TRC contingency to produce behavioral suppression in the shock baseline condition, where it seems much less likely that subjects would have learned a discrimination between the conditioning and testing phases of the experiment.

The CS - contingency also produced different effects on the two baselines. In the no-shock condition, the CS - seemed to have little effect on leverpressing. In the testing condition with noncontingent shock, however, the Pavlovian CS - significantly increased the rate of leverpressing above the baseline level. If, as would be argued by a contingency view of conditioning, the CS - was a conditioned inhibitor of anxiety, such an increase in responding would be expected in the noncontingent shock test condition where anxiety has not been systematically extinguished to situational cues during reacquisition and testing. These latter results also support and extend the findings of Hammond $(1967,1968)$ showing the inhibition of fear by Pavlovian CS - s.

A possible alternative interpretation of the differential effects of the TRC contingency on these two different baselines might be that the shock condition somehow produced a "ceiling effect." If, in fact, the TRC stimuli were mildly excitatory through their chance pairings with the aversive US, they might not have produced a suppression effect in the shock baseline, because the anxiety during testing was already at some "ceiling" level. Such an interpretation might account for the different effects of the TRC contingency across the two testing conditions; however, a possible ceiling effect does not seem able to account for the different effects of the Pavlovian $\mathrm{CS}-\mathrm{s}$ in these two testing conditions.

\section{EXPERIMENT II}

To help rule out such a "ceiling effect" interpretation and to extend the results of the first experiment, a second experiment was conducted which compared the effects of TRC stimuli and Pavlovian CS $+s$ on food-reinforced baselines with and without noncontingent shock. If noncontingent shock during testing reduced the potential excitatory effects of TRC stimuli, it should do the same for a stimulus which has had a positive contingency with the aversive US.
The two TRC conditions (tested with and without shock) were identical to those in the first experiment. The two Pavlovian CS + conditions both received the same number and percentage of CS-US pairings as the TRC condition, but had no aversive USs presented during the non-CS periods.

\section{Method}

\section{Subjects and Apparatus}

The subjects were 24 naive male Long-Evans rats, approximately 100 days old at the beginning of the experiment. The apparatus, stimuli, and shock USs were identical to those used in the first experiment.

\section{Procedure}

The experiment was a 2 by 2 factorial, consisting of two types of Pavlovian contingency during the conditioning phase (CS + and TRC) and two different reacquisition and testing conditions (with and without noncontingent shock).

Preliminary training was identical to that in the first experiment, and the Pavlovian conditioning phase was the same for the TRC subjects. The 12 subjects in the TRC condition received 15 presentations of the 1-min CS and 15 independently programmed USs. As in the first experiment, the probability of a joint occurrence of the CS and US in the TRC condition was 0.375 .

The 12 subjects in the CS + condition had exactly the same schedule of CS and US presentations as did the TRC subjects, except that all of the shock USs which would have occurred during non-CS periods were not delivered. Therefore, while both the $\mathrm{CS}+$ and TRC contingencies had four to seven CS-US pairings per session, the CS + condition had no US presentations during non-CS periods, resulting in a positive contingency.

Reacquisition and testing phases were identical to those for the first experiment Subjects in both the TRC and CS + conditions were randomly subdivided into two reacquisition and testing conditions - with and without shock. As in the first experiment, the subjects were given 3 days of reacquisition and 4 days of suppression testing.

\section{Results and Discussion}

As in the first experiment, the noncontingent shock during the final 2 days of reacquisition had the effect of preventing the complete recovery of leverpressing. The 12 subjects in the no-shock condition recovered to $91 \%$ of their preconditioning level, while subjects in the shock condition recovered to only $53 \%$ of their preconditioning level.

The effects of the TRC stimuli and Pavlovian $\mathrm{CS}+\mathrm{s}$ on the two baselines are presented in Figure 2 . As is apparent from Figure 2, the stimuli had different effects on responding as a result of the treatment conditions. A two-way repeated measures ANOVA (Treatment Conditions by Test Sessions) yielded a statistically significant effect for the Treatment Conditions $(F=9.54, \mathrm{df}=2 / 20, \mathrm{p}<.01)$. There was also a general decline in the response suppressing effects of the stimuli over test sessions, as revealed by a significant Sessions effect $(F=10.47, \mathrm{df}=7 / 140$, $\mathrm{p}<.01)$. The Treatment by Sessions interaction was not statistically significant.

As in the initial experiment, the TRC contingency in the no-shock testing condition produced a suppression effect, while the same contingency in the 


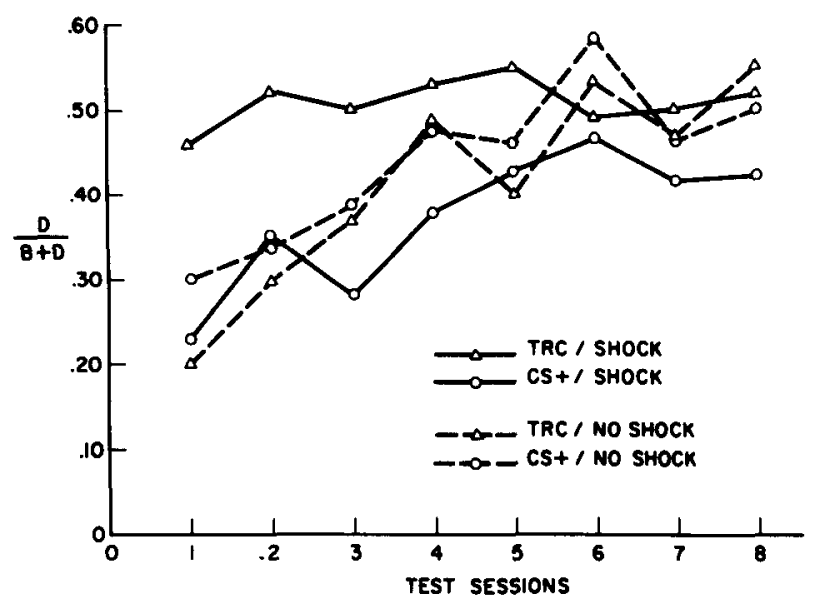

Figure 2. Mean suppression ratios for each test trial for each group in Experiment II.

shock baseline condition did not. An individual contrast on these two group means, across the eight test trials, yielded an $F=13.55(\mathrm{df}=1 / 20$, $\mathrm{p}<.01)$. The CS + contingency was not, however, affected in the same way by the type of baseline during testing. Adding noncontingent shock to the baseline did not reduce the suppression effect of the $\mathrm{CS}+$ as compared to the no-shock baseline condition. The difference between the two CS + conditions failed to reach statistical significance.

Finally, comparing the effects of $\mathrm{CS}+$ and TRC contingencies within each of the two baselines, the $\mathrm{CS}+$ contingency clearly produced a suppression effect on the shock baseline condition, while the TRC contingency did not. The difference between these two conditions produced an $F=26.58(\mathrm{df}=1 / 20$, $\mathrm{p}<.01$ ). On the no-shock baseline, however, both the CS+ and TRC contingencies produced conditioned suppression. The difference between these two conditions did not reach statistical significance.

The results from the two CS + contingencies do not seem to support a "ceiling effect" interpretation of the lack of suppression produced by the TRC contingency on the shock baseline. If, during the shock baseline condition, the failure of the TRC contingency to produce suppression was due to some type of ceiling effect, then one would expect that the same shock baseline would at least reduce the suppression effects of the CS + contingency as well. In the second experiment, while the shock baseline again eliminated suppression effects of the TRC contingency, it did not reduce the suppression produced by the $\mathrm{CS}+$.

\section{GENERAL DISCUSSION}

The two testing conditions produced clearly different patterns of results. While it is impossible to argue that one of the testing conditions shows only the effects of associative conditioning, it does seem that any experimental manipulation which increases the similarity between the conditioning and testing phases in this type of experimental paradigm is at least less likely to be influenced by nonassociative factors. There seems little question that in the testing condition with noncontingent shock, the likelihood of subjects learning a discrimination between the conditioning and testing phases of the experiment are greatly reduced by preventing the extinction of anxiety to situational cues during the reacquisition and testing.

Experiments which have reported suppression effects of TRC stimuli (e.g., Benedict \& Ayres, 1972; Kremer \& Kamin, 1971; Quinsey, 1971) used experimental procedures which seem likely to produce a discrimination between training and testing phases, and their results, therefore, could be due to nonassociative factors. By contrast, when the learning of such a discrimination is prevented or reduced, as in the present experiment's shock test condition (or in experimental paradigms using Sidman avoidance baselines, e.g., Bull \& Overmier, 1968), the influence of nonassociative factors seems less probable and TRC contingencies produce no behavioral effects.

Rescorla (1972) has suggested a different explanation of the reported suppression to TRC contingencies which he feels is consistent with a contingency view of Pavlovian conditioning. Basically, he has suggested that the excitatory effects of TRC stimuli in the above experiments is due to preasymptotic conditioning due to the relatively small number of conditioning sessions employed and/or the large proportion of the session occupied by the CS. This suggestion is not supported by the present experiments. In both experiments, the TRC contingency produced suppression in the typically used no-shock test condition in an experimental procedure where the CS occupied approximately $40 \%$ of each training session and where there was a total of 225 CS and US presentations over 15 conditioning sessions.

The effects of the CS - contingency in the noncontingent shock condition seem to provide particularly strong support for a contingency view of Pavlovian conditioning. If the noncontingent shock during reacquisition and testing did prevent extinction of anxiety to situational cues, and if the CS is a conditioned inhibitor of anxiety, the observed increase in resfonding during the CS - would be expected. Moreover, there seems to be no viable alternative explanation for this response-facilitating effect of the CS

One possible alternative interpretation of the differential effects of the TRC condition on these two baselines could be that some type of "ceiling effect" in the shock baseline condition resulted in no 
response suppression by the TRC stimulus. If this were true, then one would also have to predict that adding noncontingent shock to the baseline would reduce the suppression effect of a CS + contingency. In the second experiment, however, adding noncontingent shocks to the baseline failed to reduce response suppression to the $\mathrm{CS}+$.

One perplexing element in the results from the second experiment was the lack of a difference in the suppression effects of the CS + and TRC contingencies in the typically used no-shock test condition. This finding fails to replicate several earlier experiments reported by Rescorla (1968). The discrepancy between the present results and those of Rescorla (1968) lie not in the effects of the CS + (which produced suppression in both experiments), but rather in the effects of the TRC contingency which produced suppression in the present study but not in Rescorla's (1968) results. While the effects of the TRC contingency in the no-shock condition in the second experiment are not consistent with Rescorla's (1968) findings, they are consistent with a number of later studies (Benedict \& Ayres, 1972; Kremer \& Kamin, 1971; Quinsey, 1971) wherein suppression to TRC contingencies has been reported. Kremer and Kamin (1971) have provided some evidence that differences in the strain of rat used in these experiments may be responsible for this discrepancy.

\section{REFERENCES}

Eienedict, J. O., \& Ayres, J. B. Factors affecting conditioning in the truly random control procedure in the rat. Journal of Comparative and Physiological Psychology, 1972, 78, 323-330.

Bull, J. A., III, \& Overmier, J. B. Additive and subtractive properties of excitation and inhibition. Journal of Comparative and Physiological Psychology, 1968, 66. 511-514.
Campbell, B. A., \& Teghtsoonian, R. Electrical and behavioral effects of different types of shock stimuls on the rat. Journal of Comparative and Physiological Psychology, 1958, 51, 185-192.

Hammond, L. J. A traditional demonstration of the active properties of Pavlovian inhibition using differential CER. Psychonomic Science, 1967, 9, 65-66.

HAMMOND, L. J. Retardation of fear acquisition by a previously inhibitory CS. Journal of Comparative and Physiological Psychology, 1968, 66, 756-759.

Hoffman, H. S., \& Fleshler, M. A relay sequencing device for scrambling grid shock. Journal of the Experimental Analysis of Behavior, 1962, 5, 329-330.

HydE, T. S. The effect of Pavlovian CS+'s and CS-'s on the acquisition of a new response. Learning and Motivation. 1976, 7, 223-239.

Kremer, E. F., \& Kamin, L. J. The truly random control precedure: Associative and nonassociative effects in rats. Journal of Comparative and Physiological Psychology, 1971, 74. 203-210.

QUinseY, V. L. Conditioned suppression with no CS-US contingency in the rat. Canadian Journal of Psychology, 1971, 25, 69-82.

Rescorla, R. A. Pavlovian conditioning and its proper control procedures. Psychological Review, 1967, 74, 71-80.

Rescorla, R. A. Probability of shock in the presence and absence of $\mathrm{CS}$ in fear conditioning. Journal of Comparative and Physiological Psychology, 1968, 66, 1-5.

Rescorla, R. A. Conditioned inhibition of fear resulting from negative CS-US contingencies. Journal of Comparative and Physiological Psychology, 1969, 67, 504-509.

Rescorla, R. A. Informational variables in Pavlovian conditioning. In G. H. Bower (Ed.), The psychology of learning and motivation (Vol. 6) New York: Academic Press, 1972.

ResCORLA, R. A., \& LoLoRdo, V. M. Inhibition of avoidance behavior. Journal of Comparative and Physiological Psychology 1965, 59, 406-412.

Sheldon, M. H. Contingency theory and the distinction between associative and non-associative effects in classical conditioning. Quarterly Journal of Experimental Psychology. 1973, 25, 124-129.

(Received for publication August 5, 1974; revision received February 5, 1976.) 